Document downloaded from:

http://hdl.handle.net/10251/83063

This paper must be cited as:

Vergara Domínguez, L.; Soriano Tolosa, A.; Safont Armero, G.; Salazar Afanador, A. (2016). On the fusion of non-independent detectors. Digital Signal Processing. 50(1):24-33. doi:10.1016/j.dsp.2015.11.009.

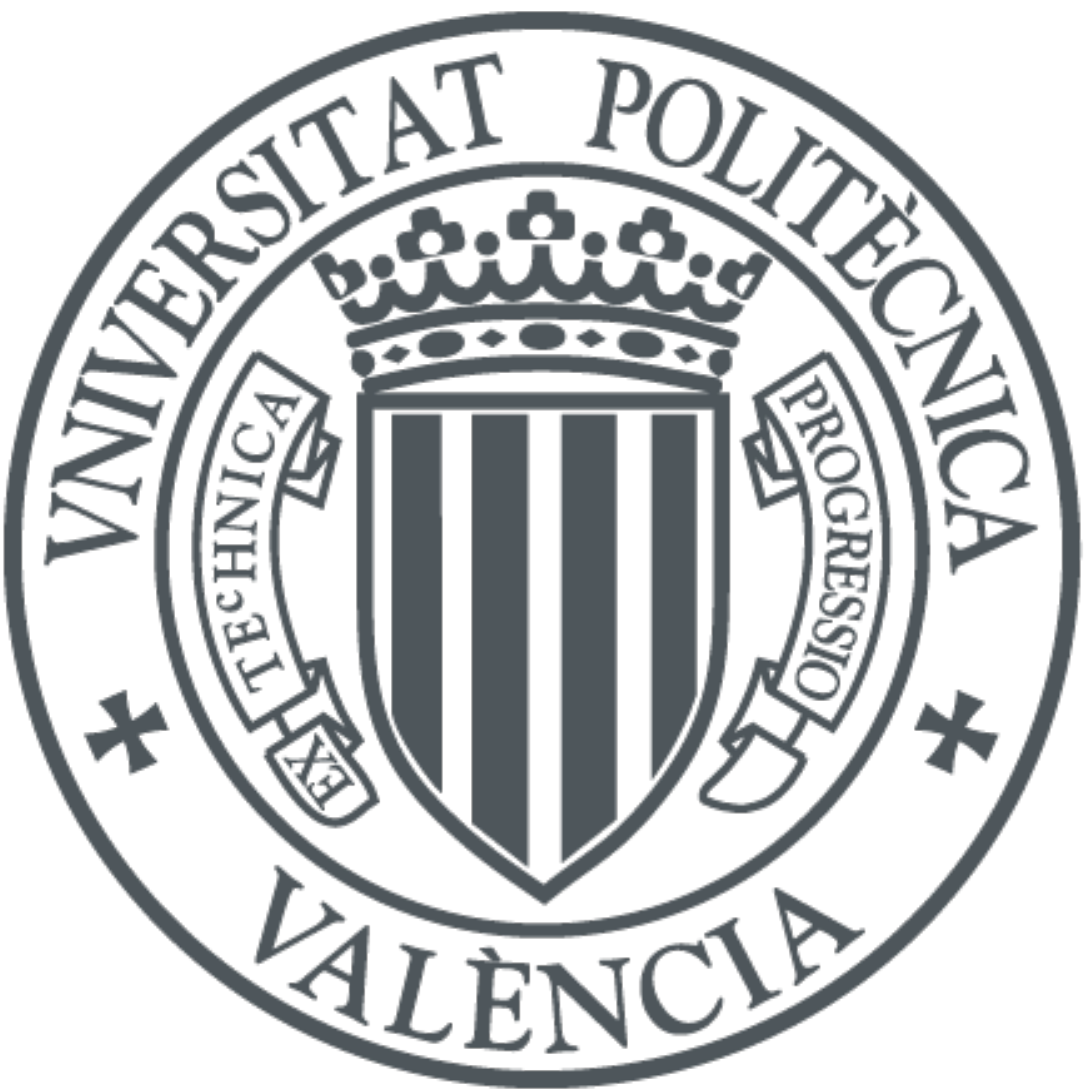

The final publication is available at

http://dx.doi.org/10.1016/j.dsp.2015.11.009

Copyright Elsevier

Additional Information 


\title{
On the fusion of non-independent detectors
}

\author{
Luis Vergara, Antonio Soriano, Gonzalo Safont, Addisson Salazar \\ Universitat Politècnica de València, Valencia, Spain
}

Corresponding author: Luis Vergara, Tel: +34963877308, Fax: +34963877309, e-mail: lvergara@dcom.upv.es

\begin{abstract}
Independence between detectors is normally assumed in order to simplify the algorithms and techniques used in decision fusion. In this paper, we derive the optimum fusion rule of $N$ non-independent detectors in terms of the individual probabilities of detection and false alarm and defined dependence factors. This has interest for the implementation of the optimum detector, the incorporation of specific dependence models and for gaining insights into the implications of dependence. This later is illustrated with a detailed analysis of the two equally-operated non-independent detectors case. We show, for example, that not any dependence model is compatible with an arbitrary point of operation of the detectors, and that optimality of the counting rule is preserved in presence of dependence if the individual detectors are "good enough". We have derived also the expressions of the probability of detection and false alarm after fusion of dependent detectors. Theoretical results are verified in a real data experiment with acoustic signals.
\end{abstract}

Keywords: Decision theory, decision fusion, dependence

\section{Introduction}

Fusion of detectors is a well-established issue, appearing in such related areas as sensor data fusion [1], multimodal fusion [2], mixture of experts [3] and classifier combiners [4]. Although frequently employing different terminologies and experiencing distinct implementation constraints, all of these areas share similar problems when considering the optimum design of fusion methods. Three different levels of fusion can be carried out: observation (in classification, "feature" is preferred) fusion, score fusion and decision fusion. In principle, observation fusion should be privileged, as it captures all the underlying statistical information about the problem. However, all the original observation components might not be 
accessible at the fusion center. This occurs in sensor networks, where transmission bandwidth conservation and distributed processing lead to only individual decisions being transmitted to the fusion center. Moreover, even given simultaneous accessibility to all the observation components, the problem of estimating the multidimensional probability densities (MPD) required for optimum, likelihood-ratiobased observation fusion, remains a complex one. This is especially the case when dealing with heterogeneous observations and/or statistical dependence between the observation vector components. Score fusion alleviates the problem of heterogeneity, as the scores afforded by each individual detector include some type of normalization: the scores are generally estimates of the a posteriori probability of every hypothesis derived from an observation. But in areas such as biometrics, scoring normalization is required prior to fusion [5]. An additional advantage garnered from score fusion is that the number of components to be fused is limited to the number of detectors. In any case, scores, like observations, are continuous variables that lend a significant degree of complexity to the task of estimating the underlying MPD, and might not be available and could not be accessible in distributed detection architectures. Consequently, decision fusion is the ideal choice when the observations or the scores are unavailable at the fusion center, and/or when MPD estimation is to be avoided or replaced by the (simpler) estimation of multidimensional probability masses (MPM).

A great deal of effort has been dedicated over the past few decades to finding optimum fusion methods at the three different levels. Some of this research, mostly recent, has assumed the presence of statistical dependence, so factoring the corresponding and underlying MPD or MPM in unidimensional marginals is not possible. In [6], a copula-based approach is therefore presented to fuse heterogeneous observations. Copulas are useful in managing heterogeneity when dependence is present: the MPD is factorized in the marginals, thereby capturing the heterogeneity, and a multidimensional copula (MPD of uniformly distributed variables derived from the original observation components) captures the dependence. Copulas are also useful in defining a variety of dependence models. Reference [7] is representative of score fusion, where the authors use non-parametric estimates of the score MPD. Optimum fusion at the decision level requires knowing the MPM of decisions made by the individual detectors. This can be obtained by multidimensional integration of the MPD in limits defined by the individual thresholds. Thus in [8], a parametric copula model (with known or unknown parameters) is assumed for the MPD, then the 
corresponding MPM are derived. In [9], the work of [8] is extended to the case of "mis-specified" copulas and multibit quantizers. Optimum design of the individual detector rules, in conjunction with the optimum definition of the decision fusion rule when dependence is present, have also been analyzed by several authors. In [10], for example, the two-sensor case is considered for the binary-quantizer Gaussian shift-in-mean problem. Determining optimal individual rules requires an iterative algorithm, where only one rule is modified at a time while the others are assumed to be fixed. Conclusions are then derived about the convergence properties of the algorithm for possible two-sensor fusion rules (AND, OR, XOR). The authors in [11] find the general expression for an optimum individual rule, given the other rules; to avoid the iterative, one-by-one search for optimal individual rules, the authors propose transforming the original observations to undo the statistical dependence and thereby render individual likelihood ratio tests optimal. Though ambitious in their quest for optimality, these works do present various drawbacks:

-It is generally assumed that the input of the individual detector is only one of the components from the entire observation vector. While this may be a reasonable assumption in distributed detection, it is not usually the case in the aforementioned related areas (see [12] for an example of fusion in the financial area), where highly dimensional patterns appear at the input of every individual detector.

-Different amounts of available information about the MPD of the observations are required or must be estimated. In [8] and [9], for example, the marginals are assumed to be known, while the copulas are known or must be estimated. In [10], Gaussianity is assumed, while in [11], the proposed transformation depends on the specific statistical model considered and may not be feasible for arbitrary scenarios.

-The algorithms are complex and mostly iterative. Hence, applying these techniques is only justified if the observations are not simultaneous available at the fusion center-not as a means of avoiding use of the MPD.

-Thus, these techniques do not generally offer an intuitive and simple understanding of the implications of dependence for the optimality of the fusion center rules. 
Simpler approaches are based on working directly with the MPM, or with equivalent representations of both the marginals and the dependence of the decisions. The optimum decision fusion rule employing the MPM is well-known (see Section IV.A of [8] and [13]). Optimum fusion rules are also proposed in [14], employing correlation coefficients of decisions and probabilities of false alarm and detection for some specific correlation structures. Based on the results from [14], [15] derives the optimal conditions for simple counting rules in the case of identical detectors, where every hypothesis is characterized by a constant correlation coefficient. Practical application of these methods requires estimating the MPM or equivalent representations. This estimation can be made from sample training records of synchronized decisions under every hypothesis.

The work presented in this paper belongs to this class of approaches. Each individual detector will be characterized by its point of operation (probability of detection PD and probability of false alarm PFA). Dependence between decisions in every hypothesis will be captured by a new defined parameters: dependence factors, DFs. These parameters are factors linking the marginal masses with the MPM in much the same way that the copula function links the marginals with the MPD, although by no means the DFs exhibit properties similar to those of copula functions [16]). We will obtain the optimum fusion rule in terms of the individuals PD and PFA and on the DFs. This facilitate the implementation of the optimum fused detector as the nature and complexity of every detector is thus of no concern, only its PD and PFA, and the DFs can be estimated from training records. Moreover, we will see that the optimum fusion rule in presence of dependence is an obvious extension of the optimum fusion rule of independent detectors. This makes possible a straightforward consideration of particular dependence models. It also allows gaining insights into the implications of dependence in different aspects such as optimality of the counting rule, detector performance or compatibility between the dependence model and the points of operation of the individual detectors.

The remainder of this paper is organized as follows. In Section 2, it is derived the optimum fusion rule in terms of the individual PD and PFA, and the DFs. Then, Section 3 focus in the two equally-operated non-independent case; this particularization is a convenient assumption for mathematical tractability, and it does not inhibit deriving interesting conclusions about the influence of the statistical dependence in the 
decision fusion problem. Section 4 provides three representative examples to illustrate the theory and a real data application to verify the theoretical predictions, with conclusions discussed in Section 5.

\section{Optimum fusion rule in terms of dependence factors}

Let us consider the case of $N$ detectors indexed by $n=1 \ldots N$. Every detector generates a binary decision $u_{n}=1,0$ and is characterized by some particular probability of detection $P_{d n}$ and probability of false alarm $P_{f n}$. We define the vector of decisions $\mathbf{u}=\left[u_{1} \ldots u_{N}\right]^{T}$. The optimum fusion rule is given by

$$
\Lambda(\boldsymbol{u})=\frac{P_{H_{1}}(\mathbf{u})}{P_{H_{0}}(\mathbf{u})} \underset{H_{0}}{>} t
$$

Where $P_{H_{j}}(\mathbf{u})$ is the MPM corresponding to hypothesis $H_{j}$ and $t$ is a threshold which determines the PD and PFA. $\Lambda(\boldsymbol{u})$ is the likelihood ratio. In the following we are going to express the optimum fusion rule in terms of the individual PD and PFA of every detector and of some properly defined dependent factors.

Let us define also the truncated vector of decisions $\mathbf{u}_{n}=\left[u_{n} \ldots u_{N}\right]^{T} n=1 \ldots N$, hence $\mathbf{u}_{1} \equiv \mathbf{u}$ and $\mathbf{u}_{N} \equiv u_{N}$. Using the probability chain rule, the MPM corresponding to the hypothesis $H_{j}$ may be expressed in the form

$$
\begin{gathered}
P_{H_{j}}(\mathbf{u})=P_{H_{j}}\left(u_{1} \mid \mathbf{u}_{2}\right) P_{H_{j}}\left(u_{2} \mid \mathbf{u}_{3}\right) \ldots P_{H_{j}}\left(u_{N-1} \mid \mathbf{u}_{N}\right) P_{H_{j}}\left(u_{N}\right)= \\
\frac{P_{H_{j}}\left(u_{1} \mid \mathbf{u}_{2}\right)}{P_{H_{j}}\left(u_{1}\right)} \frac{P_{H_{j}}\left(u_{2} \mid \mathbf{u}_{3}\right)}{P_{H_{j}}\left(u_{2}\right)} \ldots \frac{P_{H_{j}}\left(u_{N-1} \mid \mathbf{u}_{N}\right)}{P_{H_{j}}\left(u_{N-1}\right)} \prod_{n=1}^{N} P_{H_{j}}\left(u_{N}\right)=\alpha_{j}\left(u_{1} \ldots u_{N}\right) \prod_{n=1}^{N} P_{H_{j}}\left(u_{N}\right)
\end{gathered}
$$

In this way, the MPM is factorized in the marginals and in a dependence function $\alpha_{j}\left(u_{1} \ldots u_{N}\right)$ in a manner similar to that performed with a copula function [16]. Now, let the DFs under $H_{j}$ be defined by

$$
\beta_{j n}\left(\mathbf{u}_{n+1}\right)=\frac{P_{H_{j}}\left(u_{n}=1 \mid \mathbf{u}_{n+1}\right)}{P_{H_{j}}\left(u_{n}=1\right)} \quad n=1 \ldots N-1
$$

Notice that

$$
\frac{P_{H_{j}}\left(u_{n}=0 \mid \mathbf{u}_{n+1}\right)}{P_{H_{j}}\left(u_{n}=0\right)}=\frac{1-P_{H_{j}}\left(u_{n}=1 \mid \mathbf{u}_{n+1}\right)}{1-P_{H_{j}}\left(u_{n}=1\right)}=\frac{1-\beta_{j n}\left(\mathbf{u}_{n+1}\right) P_{H_{j}}\left(u_{n}=1\right)}{1-P_{H_{j}}\left(u_{n}=1\right)} .
$$


Combining (2), (3) and (4) and grouping the factors corresponding to $u_{n}=1$ in $\mathbf{u}$ separately from the factors corresponding to $u_{n}=0$, we can write

$$
P_{H_{j}}(\mathbf{u})=\prod_{n=1}^{N}\left(\beta_{j n}\left(\mathbf{u}_{n+1}\right) P_{H_{j}}\left(u_{n}=1\right)\right)^{u_{n}}\left(1-\beta_{j n}\left(\mathbf{u}_{n+1}\right) P_{H_{j}}\left(u_{n}=1\right)\right)^{1-u_{n}} \quad .
$$

Where we have defined $\beta_{j N}\left(\mathbf{u}_{N+1}\right) \equiv 1$ in (5) to include the factors corresponding to $n=N$ in a compact manner. Also notice that the PD and the PFA of sensor $n$ are respectively $P_{d n}=P_{H_{1}}\left(u_{n}=1\right)$ and $P_{f n}=P_{H_{0}}\left(u_{n}=1\right)$. Hence, using (4) we can write the expression of the likelihood ratio of (1) in terms of $P_{d n}, P_{f n}$, and the DFs.

$$
\Lambda(\mathbf{u})=\prod_{n=1}^{N}\left(\frac{\beta_{1 n}\left(\mathbf{u}_{n+1}\right) P_{d n}}{\beta_{0 n}\left(\mathbf{u}_{n+1}\right) P_{f n}}\right)^{u_{n}}\left(\frac{1-\beta_{1 n}\left(\mathbf{u}_{n+1}\right) P_{d n}}{1-\beta_{0 n}\left(\mathbf{u}_{n+1}\right) P_{f n}}\right)^{1-u_{n}}
$$

Finally, taking logarithms, the optimum fusion rule can be expressed as

$$
\sum_{n=1}^{N} u_{n} \log \left(\frac{\beta_{1 n}\left(\mathbf{u}_{n+1}\right) P_{d n}}{\beta_{0 n}\left(\mathbf{u}_{n+1}\right) P_{f n}}\right)+\left(1-u_{n}\right) \log \left(\frac{1-\beta_{1 n}\left(\mathbf{u}_{n+1}\right) P_{d n}}{1-\beta_{0 n}\left(\mathbf{u}_{n+1}\right) P_{f n}}\right) \underset{H_{0}}{\stackrel{H_{1}}{>}} \log t
$$

This is an obvious extension of the optimum fusion rule of independent detectors, in which case $P_{H_{j}}\left(u_{n}=1 \mid \mathbf{u}_{n+1}\right)=P_{H_{j}}\left(u_{n}=1\right) \quad \forall n$ and $\forall \mathbf{u}_{n+1}$, then, from the definition (3), $\beta_{1 n}\left(\mathbf{u}_{n+1}\right)=$ $\beta_{0 n}\left(\mathbf{u}_{n+1}\right)=1 \forall n$ and $\forall \mathbf{u}_{n+1}$. Substituting in (7) and rearranging terms we can express the optimum fusion rule of independent detectors in the form $\sum_{n=1}^{N} u_{n}\left(\log \left(\frac{P_{d n}\left(1-P_{f n}\right)}{P_{f n}\left(1-P_{d n}\right)}\right)\right) \underset{H_{0}}{\stackrel{H_{1}}{>}} \log \left(t\left(\frac{1-P_{f n}}{1-P_{d n}}\right)^{n}\right)$, which is a simple linear combination of the individual decisions as reported elsewhere ([13], [14] and references therein). However, in general, (7) implies the computation of a nonlinear function of the individual decisions.

Notice that the DFs in conjunction with the point of operations are a complete characterization of the MPM. Actually, the range of $P_{H_{j}}(\mathbf{u})$ is composed by $2^{N}$ (positive and less than 1) values corresponding to the $2^{N}$ values binary codes of the domain of $\mathbf{u}$. But $\sum_{\mathbf{u}} P_{H_{j}}(\mathbf{u})=1$, so we actually have $2^{N}-1$ degrees of freedom to define $P_{H_{j}}(\mathbf{u})$. Let us verify that there is the same number of degrees of freedom 
when using the DFs and the point of operations. Consider for example $H_{1}$, we have $P_{d n} n=1 \ldots N$, and $\beta_{1 n}\left(\mathbf{u}_{n+1}\right) n=1 \ldots N-1$, so, apparently we need $N+\sum_{n=1}^{N-1} 2^{N-n}=N+2^{N}-2$. But we must take into account that $\sum_{\mathbf{u}_{n+1}} P_{H_{1}}\left(u_{n} / \mathbf{u}_{n+1}\right) P_{H_{1}}\left(\mathbf{u}_{n+1}\right)=P_{H_{1}}\left(u_{n}\right)=P_{d n}$, this imposes $N-1$ constraints in the DFs

$$
\sum_{\mathbf{u}_{n+1}} \beta_{1 n}\left(\mathbf{u}_{n+1}\right) P_{H_{1}}\left(\mathbf{u}_{n+1}\right)=1 \quad n=1 \ldots N-1
$$

Hence, finally the actual number of degrees of freedom is $\left(N+2^{N}-2\right)-(N-1)=2^{N}-1$, as expected.

The same verification can be made for $H_{0}$.

The obtained optimum fusion rule (equation (7)) corresponding to the general case of $N$ differently operated non-independent detectors can be implemented after knowledge of the points of operation of the detectors $P_{d n}, P_{f n} n=1 \ldots N$, and of the DFs $\beta_{j n}\left(\mathbf{u}_{n+1}\right) j=1,0 n=1 \ldots N-1$. These later can be estimated from training records by simply counting the percentage of times that $u_{n}=1$ for every possible $\mathbf{u}_{n+1}$. Moreover, equation (6) allows straightforward consideration of particular models of dependence by properly selecting the DFs. Thus, for example, one interesting aspect of the alternative characterization of the MPM based on the DFs and the points of operation is that it is possible to reduce the number of required values for specific models of statistical dependence in a simple manner. In practical applications one may expect different degrees of dependence between different detectors. For example, if the different detectors correspond to different locations it may happens that, given one detector, only those ones which are closer exhibit some dependence with it. Similarly, in multimodal fusion, detectors of the same modality use to exhibit higher correlations than the detectors of different modalities. One typical case is biometric analysis which combines visual, audio and other types of indicators (fingerprint, iris ...). The decisions obtained from the left and right index fingerprints are much more correlated between them than with decisions obtained from other biometric indicators.

To be more specific, let us consider a simple example. We want to detect some acoustic event with 4 microphones located in the corners of a square. Let us assume that we may consider that the acoustic detector corresponding to every single microphone exhibits statistical dependence on only the two detectors corresponding to the two closer microphones, and may be considered independent of the 
remaining microphone. Using the characterization based on the DFs and the points of operation we may reduce the number of required values, as we show in the following for $H_{1}$. We have to estimate $\beta_{1 n}\left(\mathbf{u}_{n+1}\right) n=1,2,3$.

But considering the particular dependence model, it happens that, if we number the detectors clockwise as $1,2,3,4$, detector 1 is independent of detector 3 and detector 2 is independent of detector 4 . Therefore

$$
\begin{aligned}
& \beta_{11}\left(u_{2} u_{3} u_{4}\right)=\beta_{11}\left(u_{2} u_{4}\right) \rightarrow 2^{2}-1=3 \text { values required instead of } 2^{3}-1=7 \\
& \beta_{12}\left(u_{3} u_{4}\right)=\beta_{12}\left(u_{3}\right) \rightarrow 2^{1}-1=1 \text { value required instead of } 2^{2}-1=3 \\
& \beta_{13}\left(u_{4}\right) \quad \rightarrow \quad 2^{1}-1=1 \text { value required }
\end{aligned}
$$

We need also 4 more values, the points of operation, $P_{d n} n=1 \ldots 4$. So finally we need 9 values instead of 15 . Similar conclusion may be reached for $H_{0}$, therefore we need only 18 values, instead of 30 , for a complete characterization of the problem.

With the aim of gaining insights into the dependence issue, in the next section we are going to consider the simplest case of two equally operated detectors, using the presented DFs characterization of the MPM. This is a convenient assumption for mathematical tractability, and it does not inhibit deriving interesting conclusions about the influence of the statistical dependence in the decision fusion problem.

\section{Two equally-operated non-independent detectors}

\section{A. Nonlinear optimum fusion rule}

Let us consider the case of two equally operated detectors, that is $=2, P_{d 1}=P_{d 2}=P_{d} \quad P_{f 1}=P_{f 2}=$ $P_{f}$. The likelihood ratio can be obtained from (6)

$$
\Lambda\left(u_{1}, u_{2}\right)=\left(\frac{\beta_{11}\left(u_{2}\right) P_{d}}{\beta_{01}\left(u_{2}\right) P_{f}}\right)^{u_{1}}\left(\frac{1-\beta_{11}\left(u_{2}\right) P_{d}}{1-\beta_{01}\left(u_{2}\right) P_{f}}\right)^{1-u_{1}}\left(\frac{P_{d}}{P_{f}}\right)^{u_{2}}\left(\frac{1-P_{d}}{1-P_{f}}\right)^{1-u_{2}}
$$

Now, the constraint (8) takes the form

$$
\beta_{11}\left(u_{2}=1\right) P_{d}+\beta_{11}\left(u_{2}=0\right)\left(1-P_{d}\right)=1
$$

and the equivalent constraint for $H_{0}$ is

$$
\beta_{01}\left(u_{2}=1\right) P_{f}+\beta_{01}\left(u_{2}=0\right)\left(1-P_{f}\right)=1
$$


Hence, if we simply define $\beta_{11}\left(u_{2}=1\right)=\beta_{1}$ and $\beta_{01}\left(u_{2}=1\right)=\beta_{0}$, we have that $\beta_{11}\left(u_{2}=0\right)=$ $\frac{1-\beta_{1} P_{d}}{1-P_{d}}$ and $\beta_{01}\left(u_{2}=0\right)=\frac{1-\beta_{0} P_{f}}{1-P_{f}}$ and, after some straightforward elaboration, we can express the likelihood (9) in terms of the four parameters $P_{d}, P_{f}, \beta_{1}, \beta_{0}$, i.e.,

$$
\Lambda\left(u_{1}, u_{2}\right)=\left(\frac{\beta_{1} P_{d}^{2}}{\beta_{0} P_{f}^{2}}\right)^{u_{1} u_{2}}\left(\frac{P_{d}-\beta_{1} P_{d}^{2}}{P_{f}-\beta_{0} P_{f}^{2}}\right)^{\left(1-u_{1}\right) u_{2}}\left(\frac{P_{d}-\beta_{1} P_{d}^{2}}{P_{f}-\beta_{0} P_{f}^{2}}\right)^{u_{1}\left(1-u_{2}\right)}\left(\frac{1-2 P_{d}+\beta_{1} P_{d}^{2}}{1-2 P_{f}+\beta_{0} P_{f}^{2}}\right)^{\left(1-u_{1}\right)\left(1-u_{2}\right)}
$$

Grouping terms and taking logarithms we can express the optimum fusion rule in the form $u_{1} u_{2}\left(\log \frac{\beta_{1} P_{d}^{2}}{\beta_{0} P_{f}^{2}}\left(\frac{P_{f}-\beta_{0} P_{f}^{2}}{P_{d}-\beta_{1} P_{d}^{2}}\right)^{2} \frac{1-2 P_{d}+\beta_{1} P_{d}^{2}}{1-2 P_{f}+\beta_{0} P_{f}^{2}}\right)+\left(u_{1}+u_{2}\right) \log \frac{P_{d}-\beta_{1} P_{d}^{2}}{P_{f}-\beta_{0} P_{f}^{2}} \frac{1-2 P_{f}+\beta_{0} P_{f}^{2}}{1-2 P_{d} \mp P_{d}^{2}} \underset{H_{0}}{>} \log \left(t \frac{1-2 P_{f}+\beta_{0} P_{f}^{2}}{1-2 P_{d}+\beta_{1} P_{d}^{2}}\right)$

which is a nonlinear function of the individual decisions. When the detectors are independent $\beta_{1}=\beta_{0}=$ 1 and the optimum fusion rule is a linear combination of the decisions: $\left(u_{1}+u_{2}\right) \log \left(\frac{P_{d}\left(1-P_{f}\right)}{P_{f}\left(1-P_{d}\right)}\right)$ $\underset{H_{0}}{>} \log \left(t\left(\frac{1-P_{f}}{1-P_{d}}\right)^{2}\right)$

\section{B. Bounds of the dependence models}

One of the interest of the DFs characterization is that we can deduce that, given the point of operation of the detectors, not any model of dependence is possible. From (10) we have that $\beta_{11}\left(u_{2}=0\right)=\frac{1-\beta_{1} P_{d}}{1-P_{d}}$ , but, by definition $\quad 0 \leq \beta_{11}\left(u_{2}=0\right) \leq \frac{1}{P_{d}}$, hence $0 \leq \frac{1-\beta_{1} P_{d}}{1-P_{d}} \leq \frac{1}{P_{d}} \Leftrightarrow \frac{2 P_{d}-1}{P_{d}{ }^{2}} \leq \beta_{1} \leq \frac{1}{P_{d}}$, but by definition $\beta_{1}$ cannot be negative, so in conclusion (a similar reasoning could be made with $\beta_{0}$ )

$$
\max \left\{0, \frac{2 P_{f}-1}{P_{f}^{2}}\right\} \leq \beta_{0} \leq \frac{1}{P_{f}} \quad \max \left\{0, \frac{2 P_{d}-1}{P_{d}^{2}}\right\} \leq \beta_{1} \leq \frac{1}{P_{d}}
$$

Nevertheless, practical constraints, $P_{d}>0.5$ and $P_{f}<0.5$ will henceforth be assumed, yielding the actual bounds:

$$
\beta_{0 \min }=0 \leq \beta_{0} \leq \frac{1}{P_{f}}=\beta_{0 \max } \quad \beta_{1 \min }=\frac{2 P_{d}-1}{P_{d}^{2}} \leq \beta_{1} \leq \frac{1}{P_{d}}=\beta_{1 \max }
$$


We see that for reasonably good detectors $P_{d} \approx 1$ and $\beta_{1 \max } \approx \beta_{1 \min } \approx 1$, so dependence is not a significant issue under $H_{1}$. However for a good detector $P_{f} \approx 0$, and $\beta_{0 \max } \rightarrow \infty$, dependence issue can be generally important under $H_{0}$.

The obtained bounds can be expressed also in terms of the conventional form of characterizing the dependence among decisions, the correlation coefficients $\rho_{j}, j=0,1$. First, finding the relationship between both types of dependence descriptions is straightforward:

$$
\rho_{1}=\frac{E\left[u_{1} u_{2} \mid H_{1}\right]-E\left[u_{1} \mid H_{1}\right] E\left[u_{2} \mid H_{1}\right]}{\sqrt{E\left[\left(u_{1}-E\left[u_{1} \mid H_{1}\right]\right)^{2} \mid H_{1}\right]} \sqrt{E\left[\left(u_{2}-E\left[u_{2} \mid H_{1}\right]\right)^{2} \mid H_{1}\right]}}=\frac{P_{H_{1}}\left(u_{1}=1, u_{2}=1\right)-P_{d}^{2}}{P_{d}\left(1-P_{d}\right)}=\frac{\beta_{1} P_{d}-P_{d}^{2}}{P_{d}\left(1-P_{d}\right)}=\frac{P_{d}}{1-P_{d}}\left(\beta_{1}-1\right)
$$

An similarly

$$
\rho_{0}=\frac{P_{f}}{1-P_{f}}\left(\beta_{1}-1\right)
$$

Thus, independent detectors under $H_{j}$ means $\beta_{j}=1 \Leftrightarrow \rho_{j}=0$, positive dependence is given if $\beta_{j}>$ $1 \Leftrightarrow \rho_{j}>0$ and negative dependence appears when $\beta_{j}<1 \Leftrightarrow \rho_{j}<0$. Considering the above relations, the bounds expressed in (15) also imply lower and upper bounds for the correlation coefficients

$$
\rho_{0 \min }=-\frac{P_{f}}{1-P_{f}} \leq \rho_{0} \leq 1=\rho_{0 \max } \quad \rho_{1 \min }=-\frac{1-P_{d}}{P_{d}} \leq \rho_{1} \leq 1=\rho_{1 \max } .
$$

We see that the usual assumption $-1 \leq \rho_{j} \leq 1$ is not always true, as negative correlation cannot be made arbitrarily large, depending on the point of operation. Only if $P_{d}=0.5 \quad \rho_{1 \mathrm{~min}}=-1$ and similarly with $\rho_{0 \min }$. Moreover we also see that if $P_{d} \approx 1 \Rightarrow \rho_{1 \min } \approx 0$ and $\rho_{1 \max }=1$, i.e., apparently, there is a broad range of possible positive dependence. However we have seen that this is not true as in that case $\beta_{1 \text { max }} \approx \beta_{1 \text { min }} \approx 1$. So we see that DFs, apart from the advantage indicated in section 2 , offer an alternative characterization to the correlation coefficients that can better quantify the importance of the dependence issue in comparison with the independent detectors case.

\section{Optimality of the counting rule}


The counting rule decides $H_{1}$ when the number of detectors in favor of $H_{1}$ (the number of 1's in vector $\mathbf{u}$ ) is above a given threshold. This intuitive and simple rule is the optimum fusion rule in case of $N$ independent and equally operated independent detectors. However, the presence of dependence can make this rule to be suboptimum. We will illustrate this issue by considering the particular case of two equallyoperated detectors. Let us express the optimum fusion rule (13) in the form $T\left(u_{1}, u_{2}\right) \stackrel{\substack{H_{1} \\>}}{<} t^{\prime}$, then

$$
\begin{aligned}
& T(0,0)=0 \\
& \begin{aligned}
T(1,0)=T(0,1)=\log \left(\frac{P_{d}-\beta_{1} P_{d}^{2}}{P_{f}-\beta_{0} P_{f}^{2}} \frac{1-2 P_{f}+\beta_{0} P_{f}^{2}}{1-2 P_{d}+\beta_{1} P_{d}^{2}}\right) \\
T(1,1)=\log \left(\frac{\beta_{1} P_{d}^{2}}{\beta_{0} P_{f}^{2}}\left(\frac{P_{f}-\beta_{0} P_{f}^{2}}{P_{d}-\beta_{1} P_{d}^{2}}\right)^{2} \frac{1-2 P_{d}+\beta_{1} P_{d}^{2}}{1-2 P_{f}+\beta_{0} P_{f}^{2}}\right)+\log \left(\frac{P_{d}-\beta_{1} P_{d}^{2}}{P_{f}-\beta_{0} P_{f}^{2}} \frac{1-2 P_{f}+\beta_{0} P_{f}^{2}}{1-2 P_{d}+\beta_{1} P_{d}^{2}}\right)^{2}= \\
=\log \left(\frac{\beta_{1} P_{d}^{2}}{\beta_{0} P_{f}^{2}} \frac{1-2 P_{f}+\beta_{0} P_{f}^{2}}{1-2 P_{d}+\beta_{1} P_{d}^{2}}\right)
\end{aligned}
\end{aligned}
$$

The counting rule will be optimal if $T\left(u_{1}, u_{2}\right)$ increases monotonically with the number of 1 's, i.e., if $T(0,0)<T(1,0)<T(1,1)$. Notice that by assuming the practical constraint of $P_{d}>0.5$ and $P_{f}<0.5$ $\Rightarrow \beta_{1} P_{d}^{2}>1-2 P_{d}+\beta_{1} P_{d}^{2}$ and $\beta_{0} P_{f}^{2}<1-2 P_{f}+\beta_{0} P_{f}^{2}$, so that $T(1,1)>0=T(0,0)$. However, it may happens that $T(1,0)<T(0,0)$ or $T(1,0)>T(1,1)$ depending on the values $P_{d}, P_{f}, \beta_{0}, \beta_{1}$. So we have three possible cases:

$$
\begin{aligned}
& \text { Case1: } T(0,0)<T(1,0)<T(1,1) \\
& \text { Case2: } T(1,0)<T(0,0)<T(1,1) \\
& \text { Case3: } T(0,0)<T(1,1)<T(1,0)
\end{aligned}
$$

Case 1 is the counting rule. Case 2 may appear when the dependence between the decisions of the two detectors under $H_{1}$ is significantly greater than the dependence under $H_{0}$, so that the LR is greater when both detectors decide the same (independent of what they are deciding) than when both have different decisions. Finally, Case 3 corresponds to the opposite situation: the dependence between the decisions of the two detectors under $H_{0}$ is significantly greater than the dependence under $H_{1}$, so that the LR could be greater when both detectors produce different decisions than when they decide the same (independent of 
what they are deciding). The transitions between Case1 and Case2, and between Case1 and Case 3 in (20) are given, respectively, by the conditions

$$
\begin{gathered}
\frac{\left(1-2 P_{d}+P_{d}^{2} \beta_{1}\right)}{\left(1-2 P_{f}+P_{f}^{2} \beta_{0}\right)}=\frac{P_{d}-P_{d}^{2} \beta_{1}}{P_{f}-P_{f}^{2} \beta_{0}} \rightarrow \beta_{1}=\frac{P_{f}^{2} \cdot\left(1-P_{d}\right)}{P_{d}^{2} \cdot\left(1-P_{f}\right)} \beta_{0}+\frac{P_{d}-P_{f}}{P_{d}^{2} \cdot\left(1-P_{f}\right)} \\
\frac{P_{d}-P_{d}^{2} \beta_{1}}{P_{f}-P_{f}^{2} \beta_{0}}=\frac{P_{d}^{2} \beta_{1}}{P_{f}^{2} \beta_{0}} \rightarrow \beta_{1}=\frac{P_{f}}{P_{d}} \beta_{0}
\end{gathered}
$$

Which defines two straight lines in a plane $\beta_{0}-\beta_{1}$ as depicted in figure 1 . Then the bounds of the DFs can be obtained.

$$
\begin{aligned}
& \text { Case } 1: \forall \beta_{0} \text { and } \max \left\{c, \beta_{1 \text { min }}\right\}<\beta_{1}<b \\
& \text { Case } 2: \forall \beta_{0} \text { and } b<\beta_{1} \\
& \text { Case } 3: a<\beta_{0} \text { and } \beta_{1}<c,
\end{aligned}
$$

Where

$$
\begin{gathered}
a=\frac{P_{d}}{P_{f}} \beta_{1 \min } \\
b=\frac{P_{f}^{2} \cdot\left(1-P_{d}\right)}{P_{d}^{2} \cdot\left(1-P_{f}\right)} \beta_{0}+\frac{P_{d}-P_{f}}{P_{d}^{2} \cdot\left(1-P_{f}\right)} \\
c=\frac{P_{f}}{P_{d}} \beta_{0}
\end{gathered}
$$

Figure 2 reveals how the three regions change with the operating parameters $P_{d}$ and $P_{f}$ of the individual detectors. If detectors have very good properties for detection, $P_{d} \approx 1, \beta_{1 \min } \rightarrow 1$ and $a \rightarrow \frac{1}{P_{f}}$, and Case 3 tends to disappear. If they have very good properties for false alarms, $P_{f} \approx 0, b \rightarrow \frac{1}{P_{d}}$, and Case 2 tends to disappear. Given very good individual detectors $\left(P_{d} \approx 1 \quad P_{f} \approx 0\right), a \rightarrow+\infty b \rightarrow \frac{1}{P_{d}}$, hence Case 2 and Case 3 tend to disappear and Case 1 predominates for any degree of dependence. So, in conclusion, the range of DFs for which the counting rule keeps optimal increases with the performance of the individual detectors.

D. Probability of detection and probability of false alarm after fusion 
Considering (20), there are only four possible fusion rules.

Rule 1: Decide $H_{1}$ if both detectors decide $H_{1}$

Rule 2: Decide $H_{1}$ if at least one detector decide $H_{1}$

Rule 3: Decide $H_{1}$ if both detectors decide the same hypothesis

Rule 4: Decide $H_{1}$ if both detectors decide a different hypothesis

We can see from (20) that only two Rules are optimum for every Case. Thus Rule 1 and Rule 2 are optimum in Case 1, Rule 1 and Rule 3 are optimum in Case 2, and Rule 2 and Rule 4 are optimum in Case 3. This does not prevent the use of every Rule in every Case. Notice that Rule 1 and Rule 2 are particular cases of counting rules. We have derived in the Appendix A the expressions of the PD and PFA after fusion, respectively named $P_{d g}$ and $P_{f_{g}}$, as functions of $P_{d}, P_{f}, \beta_{0}, \beta_{1}$ :

$$
\begin{array}{ll}
\text { Rule } 1 \rightarrow P_{d_{g}}=P_{d}{ }^{2} \beta_{1} & P_{f_{g}}=P_{f}^{2} \beta_{0} \\
\text { Rule } 2 \rightarrow P_{d_{g}}=2 P_{d}-P_{d}{ }^{2} \beta_{1} & P_{f_{g}}=2 P_{f}-P_{f}^{2} \beta_{0} \\
\text { Rule } 3 \rightarrow P_{d_{g}}=1-2 P_{d}+2 P_{d}{ }^{2} \beta_{1} & P_{f_{g}}=1-2 P_{f}+2 P_{f}^{2} \beta_{0} \\
\text { Rule } 4 \rightarrow P_{d_{g}}=2 P_{d}-2 P_{d}{ }^{2} \beta_{1} & P_{f_{g}}=2 P_{f}-2 P_{f}^{2} \beta_{0}
\end{array}
$$

Figures 3 to 6 represent the dependence of $P_{d g}$ and $P_{f}$, respectively, on $\beta_{1}$ and $\beta_{0}$, together with the references $P_{d}$ and $P_{f}$. This offer a simple mean of evaluating whether or not the fusion improves the individual performance of the detectors. A principal and general conclusion to be drawn is that statistical dependence plays a significant role in the final behaviour of the fused detectors. In fact, it is the combined effect of the operating point of the individual detectors $\left(P_{d}\right.$ and $\left.P_{f}\right)$ and the statistical dependence under both hypotheses $\left(\beta_{0}\right.$ and $\left.\beta_{1}\right)$ that establishes the possible benefits of fusion. More specific conclusions may be derived from the analysis of the four different fusion rules. Main conclusions are summarized in Table 1. As we can see, presence of statistical dependence may significantly vary the performance of the fused detector. Thus, maximum positive dependence under both hypothesis, i.e. $\quad \beta_{1}=\frac{1}{P_{d}}$ and $\quad \beta_{0}=\frac{1}{P_{f}}$ , makes the fused detector to behave as one unique detector in Rule 1 and Rule 2, meanwhile Rule 3 and Rule 4 becomes useless ( $H_{1}$ is always decided in Rule 3 and never decided in Rule 4). Moreover, it is 
clear that a simultaneous increase of $P_{d_{g}}$ and decrease of $P_{f_{g}}$ is not possible with Rule 1 and Rule 2; it is only possible in Rule 3 and Rule 4, but some conditions must be established regarding the value of the operating point of the individual detectors and the DFs. Thus, from it can be easily deduced that simultaneous increase of $P_{d g}$ and decrease of $P_{f_{g}}$ is possible using Rule 3 if (see figure 5) $P_{f}>1 / 3$, $\beta_{0}<\frac{3 P_{f}-1}{2 P_{f}^{2}}, \beta_{1}>\frac{3 P_{d}-1}{2 P_{d}^{2}}$ and using Rule 4 if (see figure 6) $P_{d}<2 / 3, \beta_{0}>\frac{1}{2 P_{f}}, \beta_{1}<\frac{1}{2 P_{d}}$. Loosely speaking, the detectors should be "poor" and the dependence model must be significantly different among both hypothesis. This is confirmed in the last column of Table 1 where we indicate the most suitable scenarios for every Rule. As expected the non-intuitive rules (non-counting rules) 3 and 4, are only appropriate if dependence is very different under every hypothesis. Nevertheless, performance improvements are possible with all four Rules, with improvements in one of the two parameters (increasing of probability of detection or decreasing of probability of false alarm) without significant deterioration in the other.

\begin{tabular}{|c|c|c|c|}
\hline Fusion Rule & $\begin{array}{c}\text { Probabilities after } \\
\text { fusion }\end{array}$ & Result of fusion & $\begin{array}{c}\text { Most suitable } \\
\text { scenarios }\end{array}$ \\
\hline $\begin{array}{l}\text { Rule 1: Decide } H_{1} \text { if } \\
\text { both detectors decide } \\
H_{1}\end{array}$ & $\begin{array}{l}P_{d_{g}}=P_{d}^{2} \beta_{1} \\
P_{f_{g}}=P_{f}^{2} \beta_{0}\end{array}$ & $\begin{array}{l}P_{d_{g}} \leq P_{d} \\
P_{f_{g}} \leq P_{f}\end{array}$ & $\begin{array}{c}P_{d} \cong 1 \beta_{1} \cong \frac{1}{P_{d}}, P_{d_{g}} \cong P_{d} \\
\beta_{0} \cong 0 \quad P_{f_{g}} \cong 0\end{array}$ \\
\hline $\begin{array}{l}\text { Rule 2: Decide } H_{1} \text { if at } \\
\text { least one detector } \\
\text { decide } H_{1}\end{array}$ & $\begin{array}{l}P_{d g}=2 P_{d}-P_{d}^{2} \beta_{1} \\
P_{f_{g}}=2 P_{f}-P_{f}^{2} \beta_{0}\end{array}$ & $\begin{array}{l}P_{d_{g}} \geq P_{d} \\
P_{f_{g}} \geq P_{f}\end{array}$ & $\begin{array}{c}P_{f} \cong 0 \quad \beta_{0} \cong \frac{1}{P_{f}}, P_{f_{g}} \cong P_{f} \\
\beta_{1} \cong \frac{2 P_{d}-1}{P_{d}^{2}} \rightarrow P_{d_{g}} \cong 1\end{array}$ \\
\hline $\begin{array}{l}\text { Rule 3: Decide } H_{1} \text { if } \\
\text { both detectors decide } \\
\text { the same hypothesis }\end{array}$ & $\begin{array}{l}P_{d_{g}}=1-2 P_{d}+2 P_{d}^{2} \beta_{1} \\
P_{f_{g}}=1-2 P_{f}+2 P_{f}^{2} \beta_{0}\end{array}$ & $\begin{array}{l}P_{d_{g}}>P_{d} \leftrightarrow \beta_{1} \\
>\frac{3 P_{d}-1}{2 P_{d}^{2}} \\
P_{f_{g}}<P_{f} \leftrightarrow \beta_{0} \\
<\frac{3 P_{f}-1}{2 P_{f}^{2}}\end{array}$ & $\begin{array}{c}\beta_{0} \cong 0 \leftrightarrow P_{f}>1 / 3 \\
P_{f_{g}} \cong 1-2 P_{f} \\
\beta_{1} \cong \frac{1}{P_{d}} \rightarrow P_{d_{g}} \cong 1\end{array}$ \\
\hline $\begin{array}{l}\text { Rule 4: Decide } H_{1} \text { if } \\
\text { both detectors decide a } \\
\text { different hypothesis }\end{array}$ & $\begin{aligned} P_{d_{g}} & =2 P_{d}-2 P_{d}^{2} \beta_{1} \\
P_{f_{g}} & =2 P_{f}-2 P_{f}^{2} \beta_{0}\end{aligned}$ & $\begin{array}{c}P_{d_{g}}>P_{d} \leftrightarrow \beta_{1}<\frac{1}{2 P_{d}} \\
P_{f_{g}}<P_{f} \leftrightarrow \beta_{0}>\frac{1}{2 P_{f}}\end{array}$ & $\begin{array}{c}\beta_{1} \cong 0 \leftrightarrow P_{d}<1 / 2 \\
P_{d_{g}} \cong 2 P_{d} \\
\beta_{0} \cong \frac{1}{P_{f}} \rightarrow P_{f_{g}} \cong 0\end{array}$ \\
\hline
\end{tabular}

Table 1. - Summary of two equally-operated, non-independent detector fusion rules. 


\section{Some examples and a real data experiment}

\section{A. Some examples}

Let three operating points of the individual detectors be chosen: in the first, $P_{d}=0.55, P_{f}=0.1$; in the second, $P_{d}=0.8, P_{f}=0.4$; and in the third, $P_{d}=0.8, P_{f}=0.1$. Note that in the first example, one of the requirements in Rule 4 to obtain an improvement in detection probability is satisfied: $P_{d}<2 / 3$. In the second example, one of the requirements in Rule 3 to obtain an improvement in false-alarm probability is likewise satisfied with $P_{f}>1 / 3$. In the third example, none of these constraints are satisfied.

In order to compare the results of fusion with the individual performance of each detector, the ROC (Receiver Operating Characteristics) data have been calculated and represented in Figures 7 to 9. The expected results when detectors are considered to be independent are represented by triangles, while circles are used for the dependent scenarios. To the left of the ROCs, the selected DF locations in the $\beta_{0}$ $\beta_{1}$ plane are shown together with the areas corresponding to the three cases in (20). Two options are considered for every case (identified with a different letter), and the two possible optimum fusion Rules for every Case as indicated in Section 3D, are included, and subsequently marked 1 and 2. Finally, the point of operation of the individual detectors (the same for both) is starred.

An initial conclusion to be drawn from Figures 7 to 9 is that the performance of the fused detectors would have departed significantly from the expected results had the detectors been considered independent. This confirms the importance of considering possible detector dependencies when deriving appropriate (ideally, optimal) fusion rules. More specifically, the predictions from the previous analysis can be confirmed in each of the particular scenarios. Examining Figure 7, there is therefore only one case (h1) where, after fusion, the probability of detection increases and the probability of false alarm decreases. This corresponds to a case where Rule 4 is implemented and the required conditions $\left(P_{d}<2 / 3\right.$, $\left.\beta_{0}>\frac{1}{2 P_{f}}, \beta_{1}<\frac{1}{2 P_{d}}\right)$ are met. Additionally, there is only case $(e 2)$ in Figure 8 where, after fusion, the detection probability increases while the false-alarm probability decreases. This corresponds to a case where Rule 3 is implemented and the required conditions $\left(P_{f}>1 / 3, \beta_{0}<\frac{3 P_{f}-1}{2 P_{f}^{2}}, \beta_{1}>\frac{3 P_{d}-1}{2 P_{d}^{2}}\right)$ are met. There are no cases found in Figure 9 where both probabilities simultaneously improve, however, in some 
cases, one of the probabilities improves without almost no deterioration in the other. This also occurs in Figures 7 and 8. In all cases, both probabilities remain exempt from simultaneous deterioration.

\section{B. A real data experiment}

With the aim of assessing the validity of the theoretical analysis made in this paper, we have considered a real data experiment, corresponding to the fusion of two acoustic detectors. The signals were collected in the framework of a research project [18], where monitoring the acoustic environment was part of the procedure to define all facts influencing the museum experience of a visitor. However the research may find interest in any application where two or more microphones are intended to detect possible acoustic events in a background noise. In this experiment two omnidirectional electret condenser microphones were used. There was a distance of $10 \mathrm{~cm}$ between two microphones. Two types of recordings were made. The aim of the first one was to characterize the acoustic sounds that might be present at the far field of the visitor, this will be considered as background noises. In the second type of recordings we recorded possible acoustic events near a visitor. Combining different background noises and events from the data set previously described, we may generate a large variety of acoustic records.

To accomplish the condition of equally-operated detectors we have fitted the thresholds of the implemented detectors so that the PFA was the same for both. A value $P_{f}=0.1$ was considered in the showed experiment. Then we have selected combinations of background noises and acoustic events where the obtained PDs were very similar. An average PD was considered to define the point of operation, in this case $P_{d}=0.810$. Using this selected set of combinations, the DFs were estimated, we obtained $\beta_{1}=1,082, \beta_{0}=5,011$. Notice that this indicates that there exists dependence between both detectors under both hypotheses. Also notice that these values are inside the limits predicted by equation (15)

$$
0 \leq \beta_{0} \leq \frac{1}{0.1}=10 \quad \frac{2 * 0.81-1}{0.81^{2}}=0.945 \leq \beta_{1} \leq \frac{1}{0.81}=1.234
$$

We have implemented the four fusion rules indicated in the first column of Table 1. In Table 2 we show the values predicted by the developed analysis (using the equations of the second column of Table 1) and the actual values estimated in the experiment. We see that there is a reasonable similarity. Moreover we 
have verified also (last column of Table 2) that the expected conditions expressed in the third column of Table 1 are met by the estimated probabilities. Finally notice that it can be easily determined that in this experiment we are in Case 1, so only Rule 1 and Rule 2 (counting rules) are optimum. The other two rules are suboptimum and -at least in this experiment- very useless due to the, respectively, large PFA and low PD so obtained.

\begin{tabular}{|c|c|c|c|c|}
\hline Fusion Rule & $\begin{array}{c}\text { Predicted } \\
\text { probabilities after } \\
\text { fusion }\end{array}$ & $\begin{array}{c}\text { Estimated } \\
\text { probabilities } \\
\text { after fusion }\end{array}$ & $\begin{array}{l}\text { iAre expected conditions } \\
\text { satisfied with estimated valu }\end{array}$ & les? \\
\hline Rule 1 & $\begin{array}{l}P_{d_{g}}=0.709 \\
P_{f_{g}}=0.050\end{array}$ & $\begin{array}{l}P_{d_{g}}=0.701 \\
P_{f_{g}}=0.049\end{array}$ & $\begin{array}{ll}P_{d_{g}} \leq P_{d} & 0.701 \leq 0.810 \\
P_{f_{g}} \leq P_{f} & 0.049 \leq 0.100\end{array}$ & $\begin{array}{l}\text { Yes } \\
\text { Yes }\end{array}$ \\
\hline Rule 2 & $\begin{array}{l}P_{d_{g}}=0.910 \\
P_{f_{g}}=0.149\end{array}$ & $\begin{array}{l}P_{d_{g}}=0.902 \\
P_{f_{g}}=0.141\end{array}$ & $\begin{array}{ll}P_{d g} \geq P_{d} & 0.902 \geq 0.810 \\
P_{f_{g}} \geq P_{f} & 0.138 \geq 0.100\end{array}$ & $\begin{array}{l}\text { Yes } \\
\text { Yes }\end{array}$ \\
\hline Rule 3 & $\begin{array}{l}P_{d_{g}}=0.799 \\
P_{f_{g}}=0.900\end{array}$ & $\begin{array}{l}P_{d_{g}}=0.775 \\
P_{f_{g}}=0.867\end{array}$ & $\begin{array}{c}P_{d_{g}}>P_{d} \quad \leftrightarrow \quad \beta_{1}>\frac{3 P_{d}-1}{2 P_{d}^{2}} \\
0.775<0.810 \quad \text { because } 1.082<1.089 \\
P_{f_{g}}<P_{f} \leftrightarrow \quad \beta_{0}<\frac{3 P_{f}-}{2 P_{f}^{2}} \\
0.867>0.100 \text { because } 5.011>-35\end{array}$ & $\begin{array}{l}1 \\
\\
1 \\
\text { Yes } \\
\text { Yes }\end{array}$ \\
\hline Rule 4 & $\begin{array}{l}P_{d_{g}}=0.200 \\
P_{f_{g}}=0.099\end{array}$ & $\begin{array}{l}P_{d_{g}}=0.189 \\
P_{f_{g}}=0.090\end{array}$ & $\begin{array}{c}P_{d_{g}}>P_{d} \leftrightarrow \beta_{1}<\frac{1}{2 P_{d}} \\
0.189<0.810 \text { because } 1.082>0.617 \\
P_{f_{g}}<P_{f} \leftrightarrow \beta_{0}>\frac{1}{2 P_{f}} \\
0.090<0.100 \text { because } 5.011>5\end{array}$ & $\begin{array}{l}\text { Yes } \\
\text { Yes }\end{array}$ \\
\hline
\end{tabular}

Table 2. Results corresponding to the fusion of two acoustic detectors.

$$
P_{d}=0.810, P_{f}=0.100 \quad \beta_{1}=1,082, \beta_{0}=5,011
$$

\section{Conclusions}

The problem of optimum decision fusion in presence of statistical dependence among the individual decisions has been considered. The optimum fusion rule expressed in terms of the individual operating points (PD and PFA) and defined DFs has been derived. This facilitates the implementation of the optimum fused detectors as the nature and complexity of every detector is thus of no concern, only its PD and PFA, and the DFs can be estimated from training records. On the other hand, the obtained optimum rule is an obvious extension of the optimum fusion rule of independent detectors so that specific 
dependence models can be incorporated easily. This also makes possible gaining insights into the implications of dependence, as we have shown for the particular case of two equally-operated detectors. Thus, we have verified the nonlinearity of the optimum fusion rule, the bounds of the dependence models and the conditions required to keep the optimality of the counting rule. We have also derived the expressions for the PD and PFA after fusion, thus explicitly showing the influence of dependence in the fused detector performance.

\section{Acknowledgements}

This work has been supported by the Generalitat Valenciana (under grant PROMETEOII 2014-032), and by the European Commission (under grant FP7-270318 (ArtSense))

\section{References}

[1] B.Khaleghi, A. Khamis, F. O. Karray, S.N. Razavi, «Multisensor data fusion: A review of the stateof-the-art», Information Fusion, 14 (1) (2013), 28-44.

[2] P. Atrey, M. Hossain, A. El Saddik, M. Kankanhalli, «Multimodal fusion for multimedia analysis: a survey», Multimedia Systems 16, (2010), 345-379.

[3] S. E. Yuksel, J. N. Wilson, P. D. Gader, «Twenty Years of Mixture of Experts» IEEE Trans. On Neural Networks and Learning Systems, 23 (4) (2012), 1177-1193.

[4] J. Kittler, M. Hatef, R. P. Duin, J. Matas, «On combining classifiers», IEEE Transactions on Pattern Analysis and Machine Intelligence, 20 (3) (1998), 226-239.

[5] A. Jain, K. Nandakumar, A. Ross, «Score normalization in multimodal biometric systems», Pattern Recognition, 38 (2005), 2270-2285.

[6] S. G. Iyengar, P. K. Varshney, T. Damarla, A Parametric Copula-Based Framework for Hypothesis Testing Using Heterogeneous Data», IEEE Transactions on Signal Processing, 59, (5) (2011), 2308-2319.

[7] S. Prabhakar, A.K. Jain: «Decision-level fusion in finger-print verification», Pattern Recognition, 35 (2002), 861-874.

[8] A. Sundaresan, P. K. Varshney, N. S. Rao, «Copula-Based Fusion of Correlated Decisions», IEEE Transactions on Aerospace and Electronic Systems, 47 (1) (2011), 454-471.

[9] S. G. Iyengar, R.Niu, P. K. Varshney, «Fusing dependent decisions for hypothesis testing with heterogeneous sensors», IEEE Transactions on Signal Processing, 60 (9) (2012), 4888-4896. 
[10] P. Willett, F. Swaszek, R.S. Blum: «The good, bad and ugly: Distributed detection of a known signal in dependent Gaussian noise», IEEE Transactions on Signal Processing, 48, (12), (2000) $3266-3278$.

[11] H. Chen, B. Chen, P.K. Varshney: «A new framework for distributed detection with conditionally dependent observations», IEEE Transactions on Signal Processing, 60 (3) (2012), 1409-1419.

[12] A. Salazar, G. Safont, A. Soriano, L. Vergara, «Automatic credit card fraud detection based on nonlinear signal processing», Proceedings 46TH Annual 2012 IEEE International Carnahan Conference on Security Technology, October 2012, Boston, USA, 207-212.

[13] M. Kam, Q. Zhu, W. S. Gray, «Optimal data fusion of correlated local decisions in multiple sensor detection systems», IEEE Trans. on Aerospace and Electronic Systems 28 (3) (1992), 916-920.

[14] E. Drakopoulos, C.C. Lee, «Optimum multisensor fusion of correlated local decisions», IEEE Trans. Aerospace Electron.Systems 27 (4) (1991), 593-606.

[15] L. Vergara, «On the equivalence between likelihood ratio tests and counting rules in distributed detection with correlated sensors», Signal Processing 87 (2007), 1808-1815.

[16] R. B. Nelsen, An Introduction to Copulas, Springer, Berlin 2006.

[17] R.D. Hippenstiel, Detection Theory: Application and Digital Signal Processing, CRC Press, Boca Raton, Florida, 2002.

[18] FP7-ICT (270318) (2011). “Artsense: Augmented reality supported adaptive and personalized experience in a museum based on processing real time sensor events", http: //www.artsense.eu.

\section{Appendix A}

Let us compute the MPM for the two detectors case in terms of $P_{d}, P_{f}, \beta_{1}, \beta_{0}$ :

$$
\begin{gathered}
P_{H_{1}}(\mathbf{u}=[0,0])=P_{H_{1}}\left(u_{1}=0 / u_{2}=0\right) P_{H_{1}}\left(u_{2}=0\right)=\left(1-\beta_{11}\left(u_{2}=0\right) P_{d}\right)\left(1-P_{d}\right)= \\
=\left(1-\frac{1-\beta_{1} P_{d}}{1-P_{d}} P_{d}\right)\left(1-P_{d}\right)=1-2 P_{d}+P_{d}^{2} \beta_{1} \\
P_{H_{1}}(\mathbf{u}=[1,0])=P_{H_{1}}(\mathbf{u}=[0,1])=P_{H_{1}}\left(u_{1}=\frac{1}{u_{2}}=0\right) P_{H_{1}}\left(u_{2}=0\right)= \\
=\frac{1-\beta_{1} P_{d}}{1-P_{d}} P_{d}\left(1-P_{d}\right)=P_{d}-P_{d}^{2} \beta_{1} \\
P_{H_{1}}(\mathbf{u}=[1,1])=P_{H_{1}}\left(u_{1}=1 / u_{2}=1\right) P_{H_{1}}\left(u_{2}=1\right)=P_{d}^{2} \beta_{1}
\end{gathered}
$$

And similarly

$$
P_{H_{0}}(\mathbf{u}=[0,0])=1-2 P_{f}+P_{f}^{2} \beta_{0}
$$




$$
\begin{aligned}
& P_{H_{0}}(\mathbf{u}=[1,0])=P_{H_{1}}(\boldsymbol{u}=[0,1])=P_{f}-P_{f}^{2} \beta_{0} \\
& \quad P_{H_{0}}(\mathbf{u}=[1,0])=P_{H_{1}}(\boldsymbol{u}=[0,1])=P_{f}-P_{f}^{2} \beta_{0}
\end{aligned}
$$

Let $u_{g}$ be defined as the global decision, $P_{d g}$ as the final probability of detection after fusion and $P_{f}$ as the final probability of false alarm after fusion:

$$
P_{d_{g}}=P\left(u_{g}=1 \mid H_{1}\right)=P_{H_{1}}\left(u_{g}=1\right) \quad P_{f_{g}}=P\left(u_{g}=1 \mid H_{0}\right)=P_{H_{0}}\left(u_{g}=1\right)
$$

These final probabilities of detection and false alarm can then be set in relation to the MPM of the two individual decisions:

- $\quad$ Rule $1 \rightarrow u_{g}=1$ if $\mathbf{u}=[1,1]$

$$
\begin{aligned}
& P_{d_{g}}=P_{H_{1}}\left(u_{g}=1\right)=P_{H_{1}}(\mathbf{u}=[1,1])=P_{d}^{2} \beta_{1} \\
& P_{f_{g}}=P_{H_{0}}\left(u_{g}=1\right)=P_{H_{0}}(\mathbf{u}=[1,1])=P_{f}^{2} \beta_{0}
\end{aligned}
$$

- $\quad$ Rule $2 \rightarrow u_{g}=1$ if $\mathbf{u} \in\{[1,1],[1,0],[0,1]\}$

$$
\begin{aligned}
& P_{d_{g}}=P_{H_{1}}\left(u_{g}=1\right)=P_{H_{1}}(\mathbf{u}=[1,1])+P_{H_{1}}(\mathbf{u}=[1,0])+P_{H_{1}}(\mathbf{u}=[1,1])=2 P_{d}-P_{d}^{2} \beta_{1} \\
& P_{f_{g}}=P_{H_{0}}\left(u_{g}=1\right)=P_{H_{0}}(\mathbf{u}=[1,1])+P_{H_{0}}(\mathbf{u}=[1,0])+P_{H_{0}}(\mathbf{u}=[1,1])=2 P_{f}-P_{f}^{2} \beta_{0}
\end{aligned}
$$

- $\quad$ Rule $3 \rightarrow u_{g}=1$ if $\boldsymbol{u} \in\{[0,0],[1,1]\}$

$$
\begin{aligned}
& P_{d g}=P_{H_{1}}\left(u_{g}=1\right)=P_{H_{1}}(\mathbf{u}=[0,0])+P_{H_{1}}(\mathbf{u}=[1,1])=1-2 P_{d}+2 P_{d}^{2} \beta_{1} \\
& P_{f}=P_{H_{0}}\left(u_{g}=1\right)=P_{H_{0}}(\mathbf{u}=[0,0])+P_{H_{0}}(\mathbf{u}=[1,1])=1-2 P_{f}+2 P_{f}^{2} \beta_{0}
\end{aligned}
$$

- $\quad$ Rule $4 \rightarrow u_{g}=1$ if $\mathbf{u} \in\{[0,1],[1,0]\}$

$$
\begin{aligned}
& P_{d_{g}}=P_{H_{1}}\left(u_{g}=1\right)=P_{H_{1}}(\mathbf{u}=[0,1])+P_{H_{1}}(\mathbf{u}=[1,0])=2 P_{d}-2 P_{d}^{2} \beta_{1} \\
& P_{f_{g}}=P_{H_{0}}\left(u_{g}=1\right)=P_{H_{0}}(\mathbf{u}=[0,1])+P_{H_{0}}(\mathbf{u}=[1,0])=2 P_{f}-2 P_{f}^{2} \beta_{0}
\end{aligned}
$$




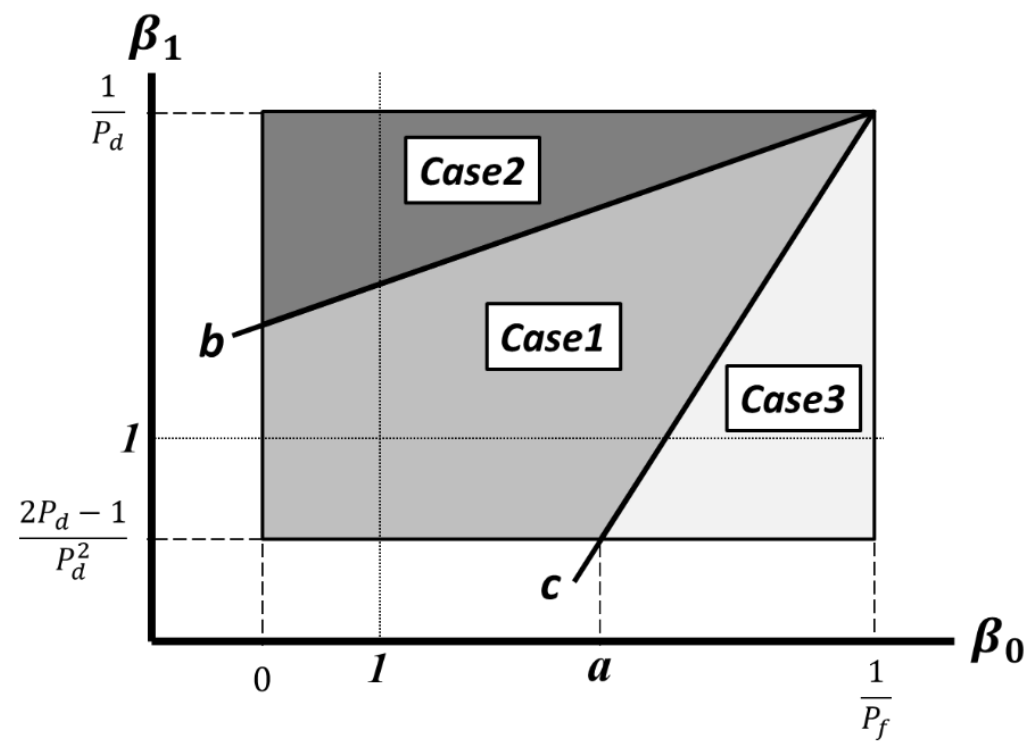

Fig. 1. Example of the three regions in the $\beta_{0} \beta_{1}$ plane, corresponding to the three possible cases defined in equation (20). 

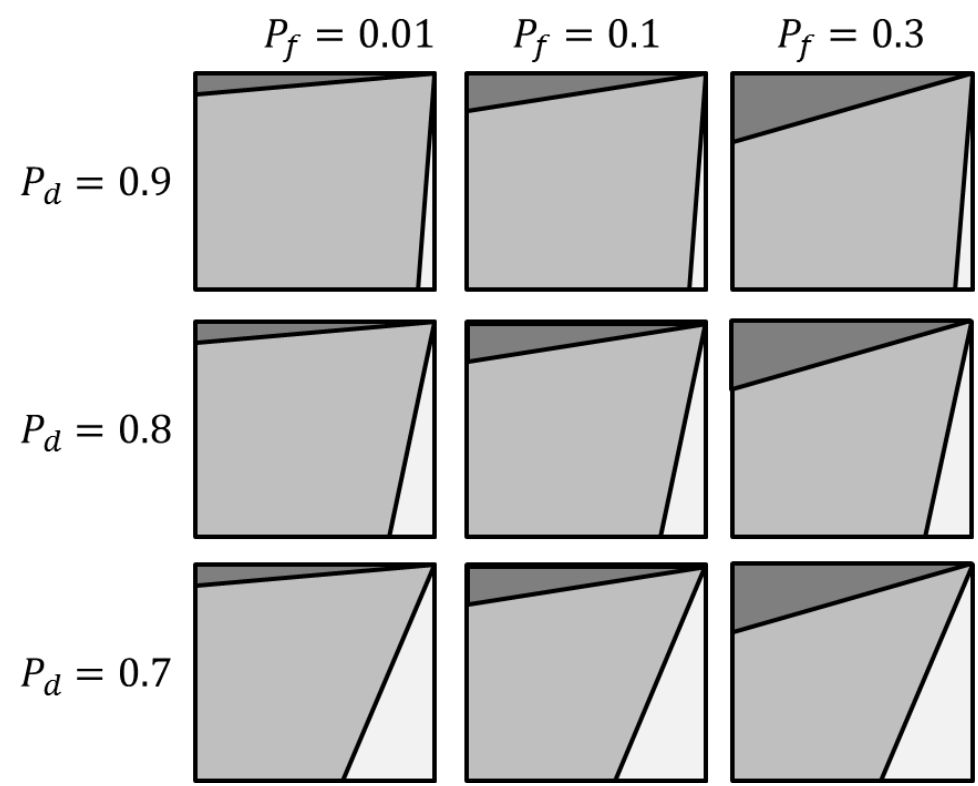

$\bigcirc \Lambda([1,0])<\Lambda([0,0])<\Lambda([1,1])$

$\bigcirc \Lambda([0,0])<\Lambda([1,0])<\Lambda([1,1])$

○ $\Lambda([0,0])<\Lambda([1,1])<\Lambda([1,0])$

Fig. 2. Variation of the three regions with the operating parameters $P_{d}$ and $P_{f}$ of the individual detectors. 

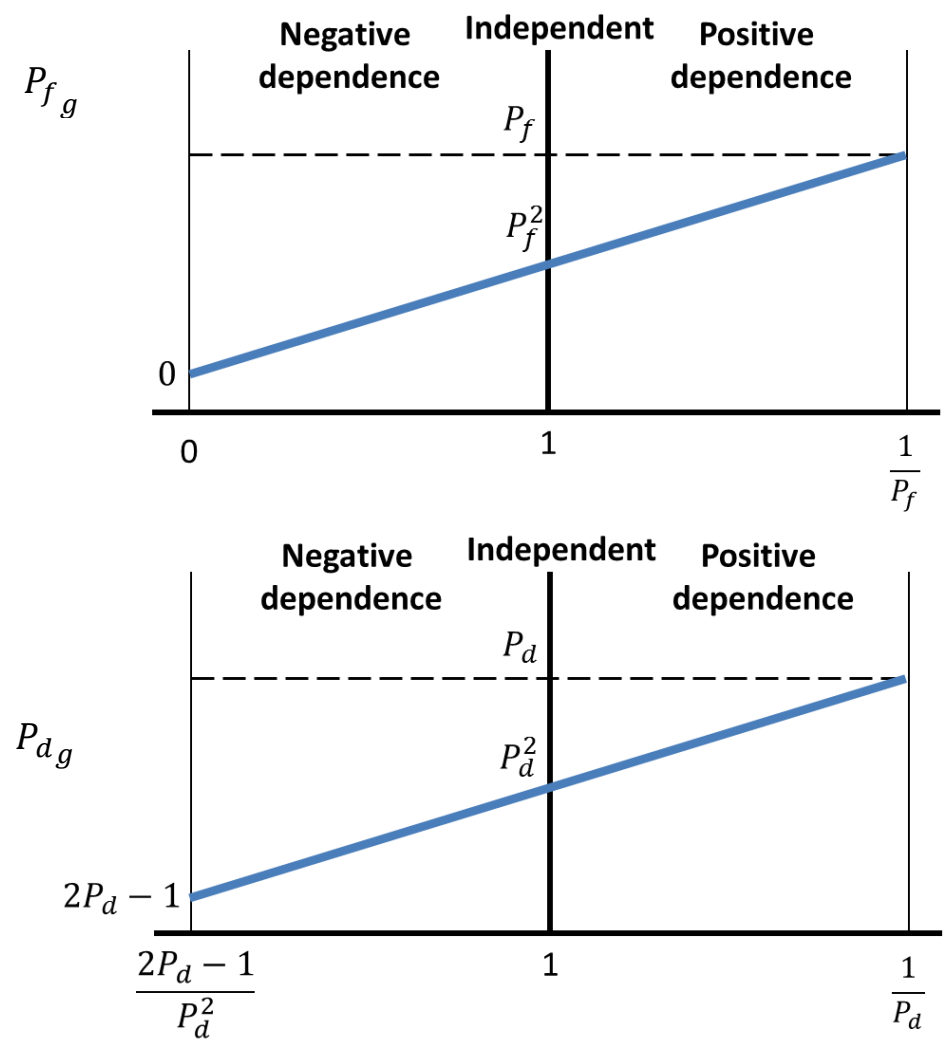

Fig. 3. Dependence of $P_{d_{g}}$ and $P_{f_{g}}$ on the DF for Rule 1. 

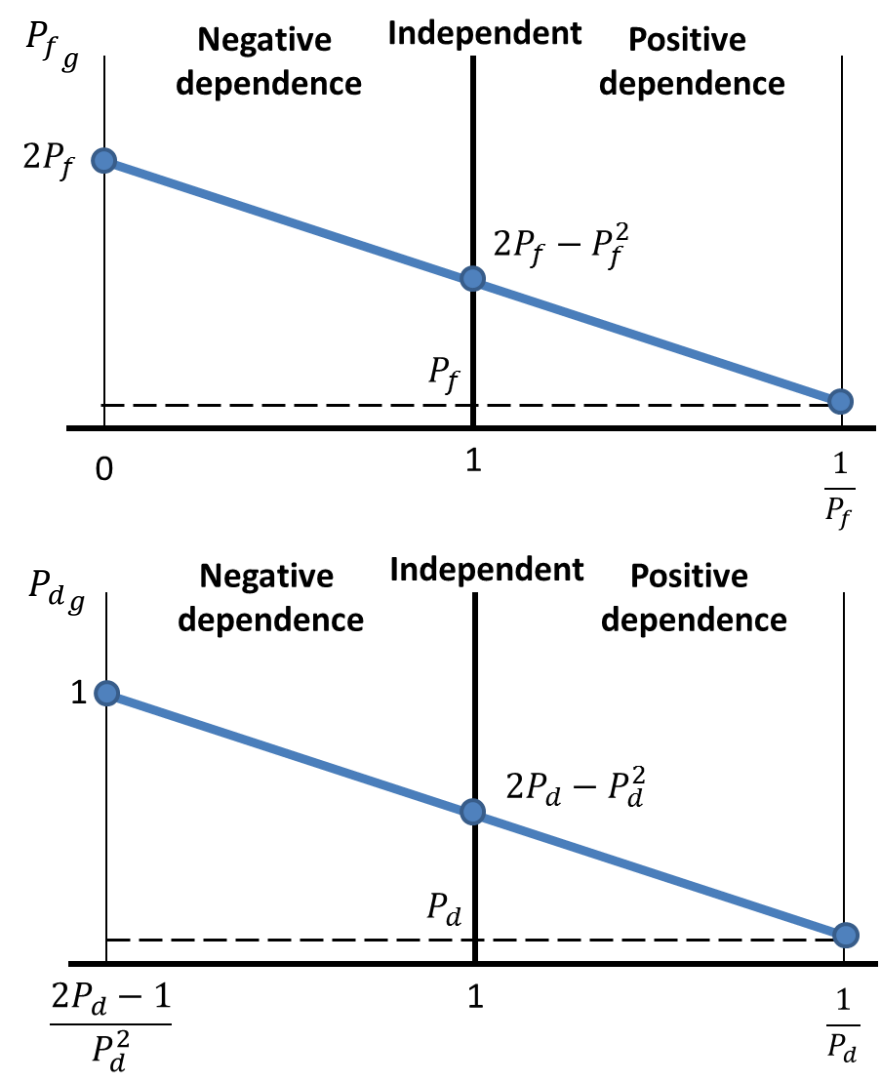

Fig. 4. Dependence of $P_{d_{g}}$ and $P_{f_{g}}$ on the DF for Rule 2. 

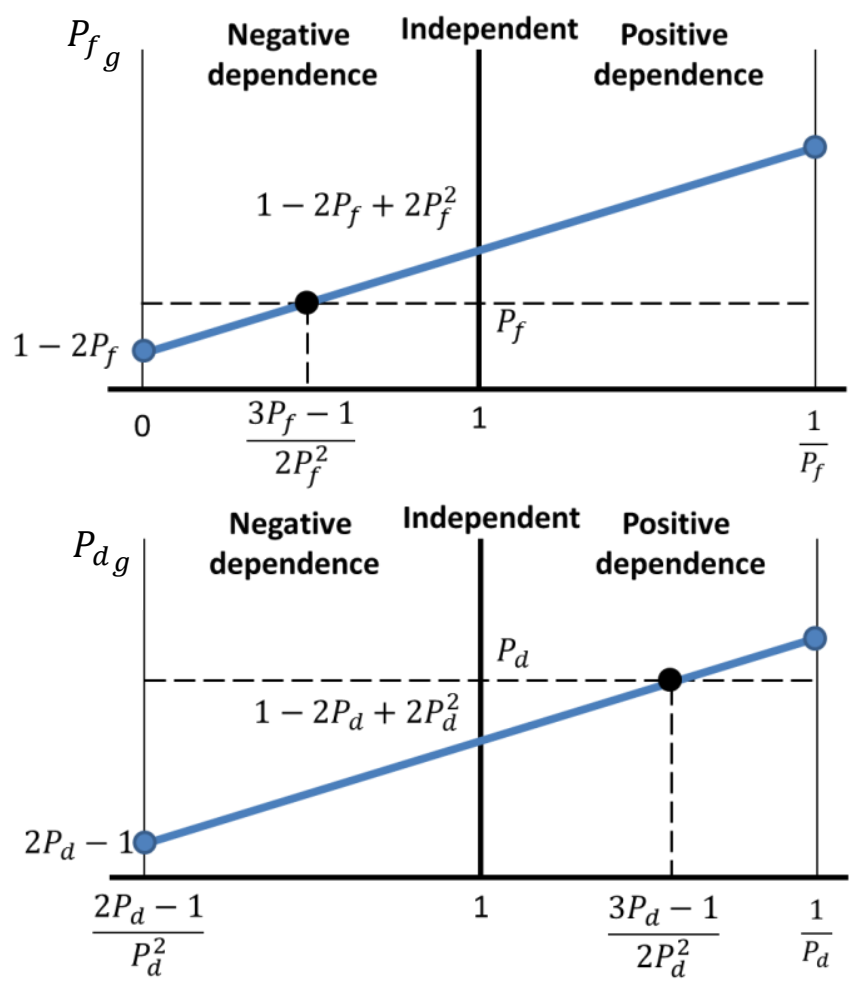

Fig.5. Dependence of $P_{d_{g}}$ and $P_{f_{g}}$ on the DF for Rule 3. 

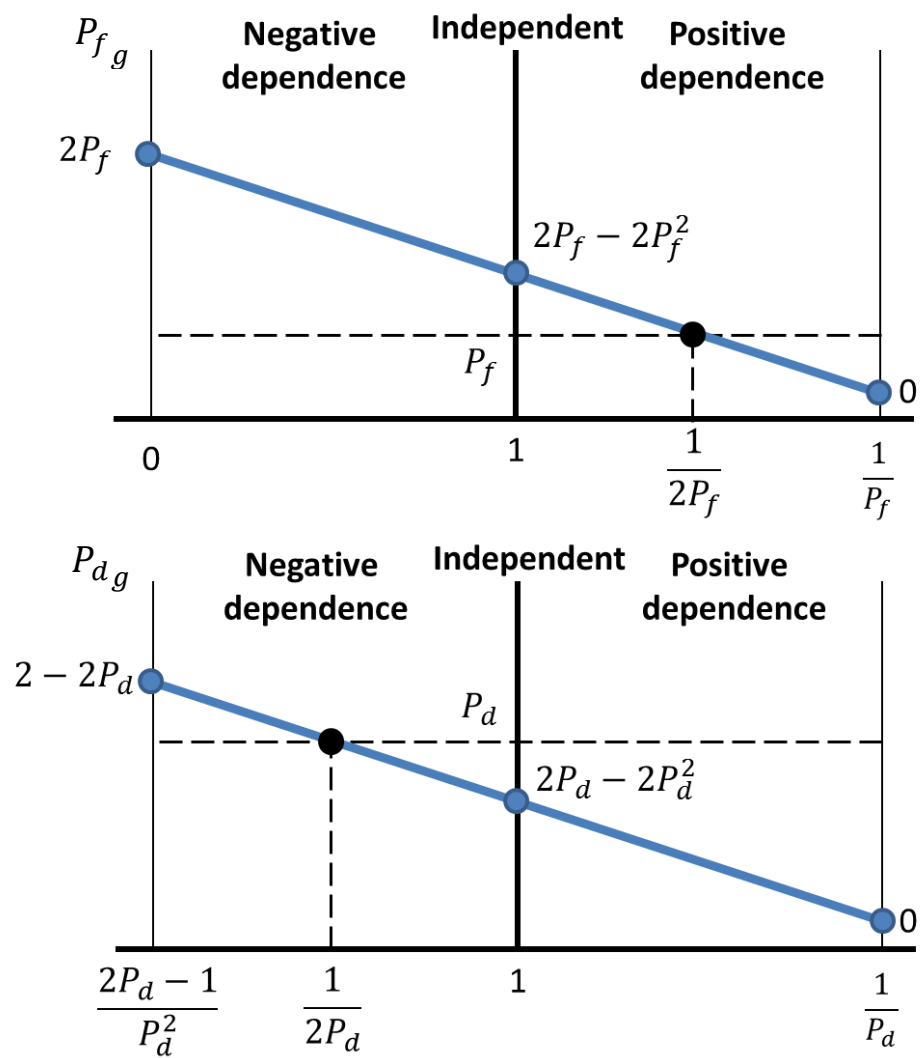

Fig. 6. Dependence of $P_{d_{g}}$ and $P_{f_{g}}$ on the DF for Rule 4 . 


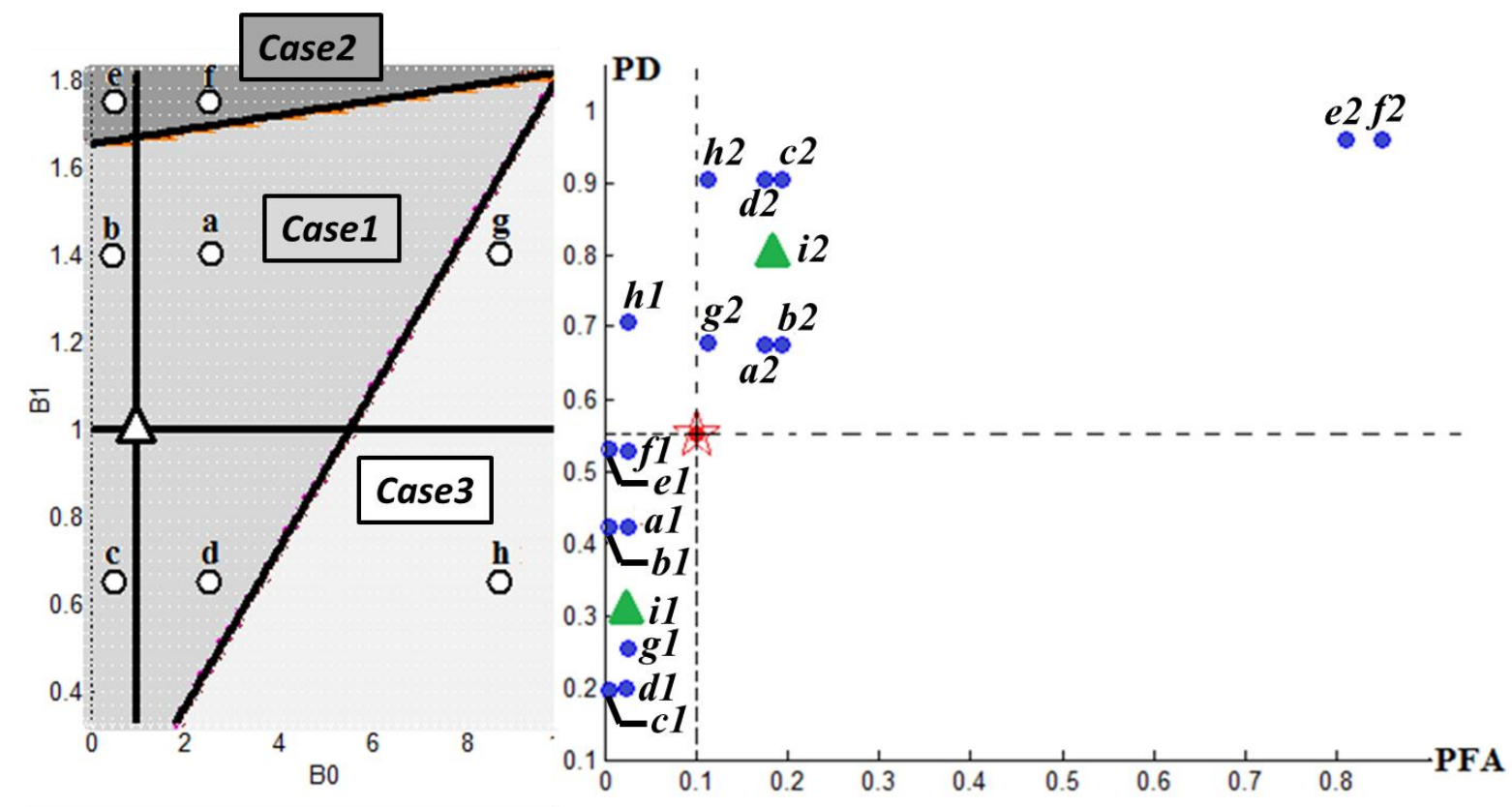

Fig. 7. ROC results corresponding to the three Cases of fusion of two non-independent detectors operating at $P_{d}=0.55$ and $P_{f}=0.1$. 


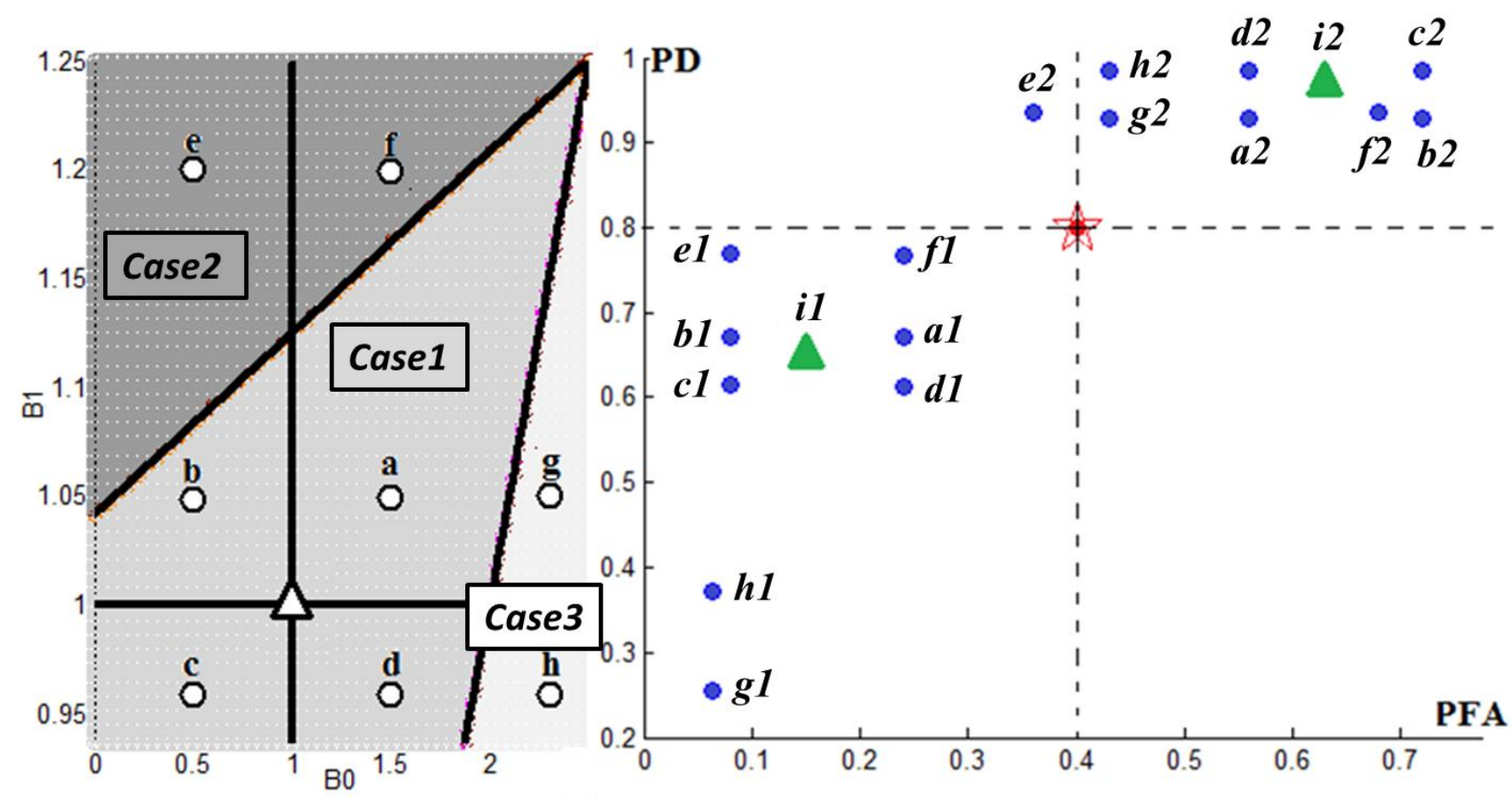

Fig. 8. ROC results corresponding to the three Cases of fusion of two non-independent detectors operating at $P_{d}=0.8$ and $P_{f}=0.4$. 


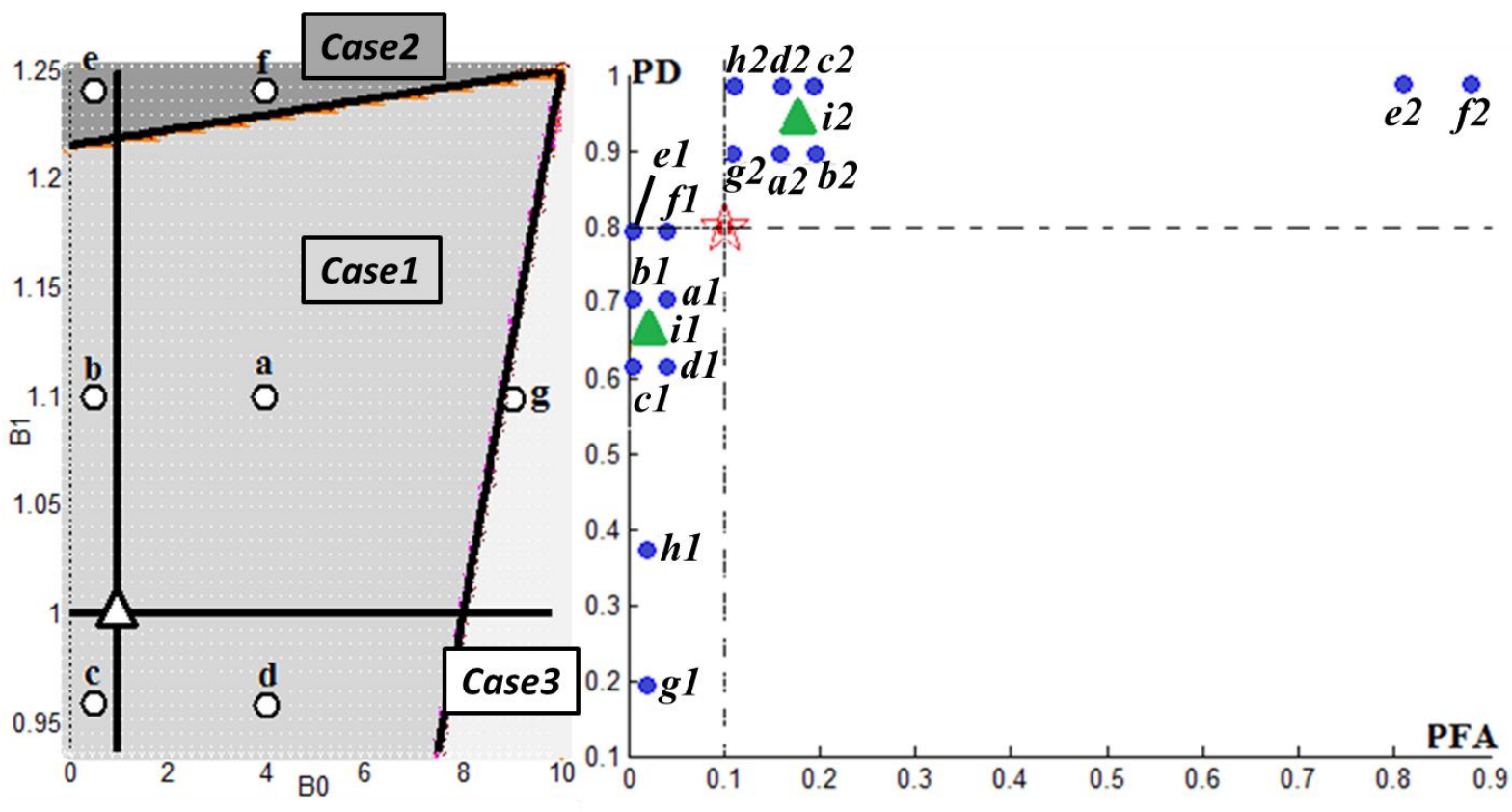

Fig. 9. ROC results corresponding to the three Cases of fusion of two non-independent detectors operating at $P_{d}=0.8$ and $P_{f}=0.1$. 